\title{
Traffic steering by self-tuning controllers in enterprise LTE femtocells
}

\author{
Jose Maria Ruiz-Avilés, Salvador Luna-Ramírez*, Matias Toril and Fernando Ruiz
}

\begin{abstract}
Femtocells have been suggested as a promising solution for the provision of indoor coverage and capacity. This article investigates the problem of re-distributing traffic demand between long-term evolution (LTE) femtocells with open access in an enterprise scenario. Several traffic sharing algorithms based on automatic tuning of femtocell parameters are considered. The proposed algorithms are implemented by fuzzy logic controllers. Performance assessment is carried out in a dynamic system-level simulator. Results show that localized congestion problems in these scenarios can be solved without impairing connection quality by jointly tuning handover margins and cell transmit power.
\end{abstract}

Keywords: Femtocell, Traffic sharing, Optimization, Handover margin, Transmit power

\section{Introduction}

Recent surveys have shown that more than $2 / 3$ of mobile traffic demand is originated at home or work, but nearly half of the houses and premises have poor indoor coverage $[1,2]$. Future cellular networks will therefore have to provide adequate indoor coverage in a cost effective manner. Femtocell access points (also known as home base stations) have been proposed as a solution for the provision of high coverage and capacity indoors. By definition, femtocells access points are low-power base stations using cellular technology in licensed frequency bands providing service indoors over internet-grade backhaul under operator management [3]. Hereafter, the terms home base station and femtocell will be interchangeably used, although the former refers to the electronic device and the latter refers to the service area of the base station.

Massive femtocell deployment for improving indoor coverage has important advantages compared to the common approach of increasing the number of macrocellular sites [4]. From the operator perspective, large operational and capital expenditures associated to conventional sites and their backhaul network are reduced. At the same time, the end user perceives a better quality of service and an increased battery lifetime due to a shorter transmission distance. Unfortunately, some difficulties also arise,

*Correspondence: sluna@ic.uma.es

Communications Engineering Dept. Málaga, University of Málaga, Málaga, Spain amongst which is the management issue. Femtocells are part of the overall radio access network and have to be managed in a coordinated way between themselves and with the rest of the network. Such a coordination is difficult because, unlike conventional sites, femtocells do not follow a careful planning by the operator due to their large number and location controlled by the user. In these conditions, self-organizing network (SON) [5] techniques play a key role in the successful deployment of femtocells.

Cell load balancing has been identified as a relevant SON use case by the industry and standardization bodies $[6,7]$. In the literature, several advanced radio resource management (RRM) algorithms can be found for handling interference and traffic in femtocells, which will be extremely valuable for manufacturers. However, to the authors' knowledge, few studies have investigated traffic sharing in enterprise femtocells with legacy equipment, which is of interest to network operators. Such scenarios have important differences with residential scenarios often covered in the literature, namely that: a) enterprise scenarios often have a three-dimensional structure, where neighbor cells are located everywhere around the serving cell, which leads to interference problems; b) a different (and probably more intense) user mobility pattern than at home; c) a higher concentration of users varying both in space (e.g., canteen) and time (e.g., arrival at work, lunch time); and d) open access instead of closed (i.e., limited) access. All these properties suggest that, in these scenarios, traffic management problems could arise and traffic

\section{是 Springer}

(C) 2012 Ruiz-Avilés et al.; licensee Springer. This is an Open Access article distributed under the terms of the Creative Commons Attribution License (http://creativecommons.org/licenses/by/2.0), which permits unrestricted use, distribution, and reproduction in any medium, provided the original work is properly cited. 
sharing is a viable means to make the most of existing femtocell resources.

This article investigates the potential of different traffic sharing techniques to solve persistent congestion problems based on network statistics in enterprise long-term evolution (LTE) femtocells. The proposed algorithms change femtocell service areas by modifying the transmit power and/or handover margins of a cell and its neighbors. For ease of development, the self-tuning algorithms are implemented by means of fuzzy logic controllers. Assessment is based on a dynamic system-level LTE simulator. The main contributions of this study are: a) a novel self-tuning method that adapts several classical traffic sharing approaches and combines them in the most effective way, and b) a thorough performance analysis of traffic sharing techniques in an extreme, albeit realistic, enterprise femtocell scenario. The rest of the article is organized as follows. Section 'Problem formulation' formulates the traffic sharing problem in enterprise femtocells. Section 'Related study' reviews the state of research and practice of cellular traffic management and femtocells. Section 'Traffic steering algorithms' outlines several traffic sharing algorithms based on tuning femtocell parameters. Section 'Performance analysis' presents simulation results and Section 'Conclusions' summarizes the main conclusions.

\section{Problem formulation}

In cellular networks, traffic sharing (or steering) aims to balance the traffic among adjacent cells in the hope that this will decrease the overall blocking ratio, thus increasing the total carried traffic in the network. To obtain such an effect, cell service areas are modified to reduce or increase traffic served by a cell. Narrowing a cell service area decreases the carried traffic in that cell by enlarging the service area of surrounding cells, provided that enough cell overlapping exists.

Re-sizing service areas can be achieved by tuning HandOver (HO) margins. The HO margin parameter from cell $i$ to cell $j$, Margin $\operatorname{PBGT}_{\text {PB }}(i, j)$, defines by how much the signal level received from a neighbor cell $j$ must exceed that of the serving cell $i$ to trigger a power budget (PBGT) HO from $i$ to $j$. Thus, a PBGT HO is triggered when

$$
\overline{\operatorname{RSRP}}(j)-\overline{\operatorname{RSRP}}(i) \geq \operatorname{Margin}_{\mathrm{PBGT}}(i, j),
$$

where $\overline{\operatorname{RSRP}}(i)$ and $\overline{\operatorname{RSRP}}(j)$ are the average reference signal received power from the serving cell $i$ and neighbor cell $j$ in $\mathrm{dBm}$, respectively, and $\operatorname{Margin}_{\mathrm{PBGT}}(i, j)$ is the margin in $\mathrm{dB}$. As observed in (1), margins are defined on an adjacency basis. Therefore, adjusting this parameter in a single adjacency only has an influence on that particular adjacency. Thus, cell service areas can not only be resized but also re-shaped. To avoid instabilities in the $\mathrm{HO}$ process, a hysteresis region can be maintained by synchronizing changes in both directions of the adjacency (i.e., if the margin from cell $i$ to $j$ is increased by $+\mathrm{X} \mathrm{dB}$, the margin from $j$ to $i$ is reduced by $-\mathrm{XdB}$ ).

The service area of a cell can also be modified by adjusting its transmit power, $P_{\mathrm{TX}}(i)$. A higher/lower transmit power in a base station is directly linked to higher/lower received signal levels in that cell, which has an influence on cell dominance areas. Unlike margins, transmit power is defined on a cell basis, so that all neighbors are equally affected by changes in the transmit power of a cell.

The modification of cell service areas also has an impact on network connection quality. As a result of traffic steering, a user might not be served by the closest base station, providing the minimum pathloss, which might impair connection quality. Although adaptive modulation and coding in LTE partly alleviates this problem, the link adaptation capability is limited. Therefore, traffic steering must be performed carefully to keep Quality-of-Service in a satisfactory level. This is important in indoor scenarios, where coverage holes and severe fading may exist due to wall obstructions and multi-path reflections. For this reason, operators often prefer to keep femtocell power settings on the default (i.e., maximum) values in the absence of precise method to predict propagation losses in indoor environment.

\section{Related study}

In cellular networks, cell service areas can be modified by different techniques. A first group of techniques adjust physical parameters in the base station, such as data or pilot transmit power [8] or antenna radiation pattern [9]. In practice, these techniques have seldom been used since they may create coverage holes (unless changes in adjacent cells are synchronized) and involve maintenance actions in legacy equipment. Alternatively, a second group of techniques change parameters in RRM processes, such as cell reselection (CR) [10] and HO [11]. Since tuning CR parameters is only effective during call set-up, the optimization of $\mathrm{HO}$ parameters is normally the preferred option. Thus, most traffic sharing algorithms rely on $\mathrm{HO}$ margins, regardless of the radio access technology (e.g., [11-17]).

To find the best margin value in each adjacency, the tuning problem can be formulated as a classical optimization problem $[18,19]$. However, as the measurements required to build the analytical model are rarely available, operators end up solving the problem by heuristic rules. An example of these is the equalization of cell traffic across the network by a diffusive load sharing algorithm. Depending on the speed of the traffic re-allocation process, the specific performance indicator to be balanced may be cell average load (e.g., [12-16]) or call blocking ratios (e.g., $[11,17])$. As shown in $[20]$, the latter option has better 
performance for persistent congestion problems due to a higher stability and, more importantly, it does not need any hardware upgrade in legacy equipment, since it can be easily included as an automatic procedure in the network management system.

Femtocells have gained attention in the research community, which is evident from several recently launched projects (e.g., HOMESNET [21], BeFEMTO [22], FREEDOM [23]). First publications were devoted to residential scenarios with standalone femtocells. For these scenarios, Claussen et al. propose in [24] a self-tuning algorithm to adjust transmit power for uplink and downlink in an Universal mobile telecommunication system (UMTS) femtocell to mitigate interference to macrocells and ensure a constant femtocell radius, regardless of the position of the latter within the macrocell area. In [25], the authors present a self-tuning algorithm for pilot power in an UMTS femtocell to improve coverage and minimize the total number of $\mathrm{HO}$ attempts. For the same purpose, [26] presents a self-tuning algorithm for selecting femtocell pilot power and antenna pattern, while [27] presents an adaptive algorithm for selecting the hysteresis margin based on user position. More recent studies have considered networked femtocell environments, among which is the enterprise scenario [28]. In these scenarios, most efforts have been paid to the design of advanced RRM algorithms to manage inter-cell interference in orthogonal frequency division multiple access (OFDMA) schemes [29]. López et al. [30] propose an integer linear programming model to dynamically assign modulation and coding scheme, radio bearer and transmit power to users, while minimizing the total cell transmit power and meeting user throughput demands. Similarly, several distributed admission control and scheduling schemes have been inspired in self-organizing principles taken from cognitive networks [31], machine learning [32,33] and game theory [34]. More related to the study presented here, dealing with self-optimization, [35] proposes a decentralized algorithm for tuning pilot power in UMTS femtocells to balance cell load and minimize total pilot transmit power in an office scenario. In [36], the problem of power and frequency planning in mobile wireless interoperability for microwave access (WiMAX) enterprise femtocells is formulated as a mixed integer programming model, whose goal can be either to maximize the sum of transmit power, given that the overall connection quality impairment is kept within acceptable limits, or to maximize network Shannon capacity.

A wide range of analysis tools have been used to evaluate the performance of femtocell networks. When mobility issues are important, dynamic system-level simulators are used. In particular, $[37,38]$ describe dynamic system-level simulators with LTE femtocells similar to the one used in this study [39].

\section{Traffic steering algorithms}

Classical load balancing algorithms conceived for GSM or UMTS can be adapted for traffic steering in an LTE enterprise scenario. The aim of the methods considered here is to solve localized and persistent congestion problems by equalizing call blocking ratio throughout the network. All methods are based on tuning two femtocell parameters: $\mathrm{HO}$ margin and transmit power.

Parameter tuning is carried out periodically by controllers. One controller per adjacency is needed to adjust $\mathrm{HO}$ margins, whereas one controller per cell is used to adjust transmit power. The decision of a parameter change is based on performance statistics and parameter settings in the previous period. Specifically, the algorithms try to minimize the difference in call blocking ratio between adjacent cells by a diffusive load balancing algorithm. Therefore, equilibrium will not be reached until such a difference in blocking ratio is negligible. Since the goal is to solve persistent congestion problems, and not temporary traffic fluctuations, input statistics are collected during a long period (i.e., above $15 \mathrm{~min}$ ).

The basic algorithms considered are:

1) Margin traffic sharing (MTS). An MTS controller modifies PBGT HO margins on a per-adjacency basis. The aim is to balance the call blocking ratio between the source and target cell of the adjacency. Changes of the same amplitude and opposite sign are performed in the margins of both directions of the adjacency to maintain cell overlapping, i.e.,

$$
\operatorname{Margin}_{\mathrm{PBGT}}(i, j)+\operatorname{Margin}_{\mathrm{PBGT}}(j, i)=\text { Hyst },
$$

where Hyst is a constant defining the hysteresis value. In this study, Hyst $=6 \mathrm{~dB}$ and the default value of $\operatorname{Margin}_{\mathrm{PBGT}}(i, j)$ is $3 \mathrm{~dB} \forall i, j$.

Changes by MTS can be restricted to a limited interval to avoid connection quality problems, as will be explained later. Such a variant will be referred to as constrained MTS (MTSC).

2) Power traffic sharing (PTS). A PTS controller tunes cell transmit power on a per-cell basis to balance the call blocking ratio of a source cell against the average call blocking ratio of its neighbors. Cells start at their maximum transmit power and decrease (increase) their power if their call blocking ratio is larger (smaller) than that of their neighbors. Transmit power is limited to the maximum default value. For simplicity, no synchronization between neighbors is considered and, consequently, cell overlapping can be affected. Likewise, it is assumed that both data and pilot power are jointly tuned. Thus, traffic steering is effective not only for connected users, but also for idle users (i.e., it has an impact on both $\mathrm{CR}$ and $\mathrm{HO}$ processes). 
The previous approaches can be combined to improve the traffic sharing capability. It is obvious that executing MTS and PTS simultaneously but independently can increase both adaptation speed and final network performance. However, it will be shown later that, by coordinating both algorithms, the limits inherent to individual approaches can be overcome. Such a joint strategy gives raise to a third algorithm, namely:

3) Combined traffic sharing (CTS). A CTS controller modifies the transmit power of a cell and $\mathrm{HO}$ margins of its adjacencies. For this purpose, a CTS controller consists of one PTS controller and several MTSC controllers (one per adjacency). All controllers in a cell are executed in a coordinated way. First, MTSC modifies HO margins while PTS is disabled. Only when all MTSC controllers in that cell have reached their limits, PTS is enabled and modifies transmit power. As a result, traffic sharing is achieved with minimal deviation of transmit power from default values.

\section{Implementation of controllers}

Tuning methods have been implemented by Fuzzy Logic Controllers (FLCs) to simplify the design of the controller. FLCs [40] are expert systems described by means of "IF-THEN" rules. Due to the fact that FLCs are described in linguistic terms, it is easier to integrate previous knowledge into the controller. Thus, FLCs are especially suited when the experience of an operator is already available (as it is the case for telecommunication networks). The main difference of FLCs with conventional rule-based controllers is their capability to trigger several rules simultaneously, which leads to smoother control actions.

In this study, an incremental FLC structure is adopted, where the output of the controller is the parameter change to be added to the previous parameter value (and not the final value itself). For instance, Figure 1 shows the FLC of the MTS strategy. As observed in the figure, FLC inputs are key performance indicators (i.e., the call blocking ratio difference between adjacent cells) and current parameter values (HO margin between adjacent cells), while FLC outputs are changes in femtocell parameters (i.e., HO margin step). Not shown in the figure is the fact that the output of the controller is rounded to the nearest integer. The new margin value is computed as

$$
\begin{aligned}
\operatorname{Margin}_{\mathrm{PBGT}}^{(n+1)}(i, j)= & \operatorname{round} \operatorname{Margin}_{\mathrm{PBGT}}^{(n)}(i, j) \\
& \left.+\delta \operatorname{Margin}_{\mathrm{PBGT}}^{(n)}(i, j)\right),
\end{aligned}
$$

where $\operatorname{Margin}_{\mathrm{PBGT}}(i, j)$ is the current margin value, $\delta$ Margin $_{\mathrm{PBGT}}(i, j)$ is the suggested margin step (in $\mathrm{dB}$ ) for such an adjacency $(i, j)$ and superindex $(n)$ indicates the iteration of the optimization process.

Inside, an FLC consists of three stages: fuzzification, inference and defuzzification. In the fuzzification stage, the numerical value of each input variable is mapped into a limited set of adjectives (e.g., high, low, ...) by a membership function defining the degree with which each value of the input can be associated to that adjective. Figure 2a presents the membership functions for MTS. VN, N, Z, P, and VP stand for very negative, negative, zero, positive and very positive, respectively. Note that, unlike conventional controllers, in an FLC, a single input value can be associated to different adjectives with different degrees (and, hence, the term 'fuzzy'). In this study, the number of input membership functions has been selected large enough to classify performance indicators as precisely as an experienced operator would do, while keeping the number of states small to reduce the set of control rules. For simplicity, the selected input membership functions are trapezoidal, triangular or constant. In the inference stage, a set of 'IF-THEN' rules defines the mapping of inputs to output. Figure $2 b$ shows the rule database for MTS. For instance, rule 1 reads as "IF $\mathrm{BR}_{\text {diff }}$ is very positive THEN $\delta \operatorname{Margin}_{\mathrm{PBGT}(i, j)}$ is very negative". Roughly, the more positive (negative)

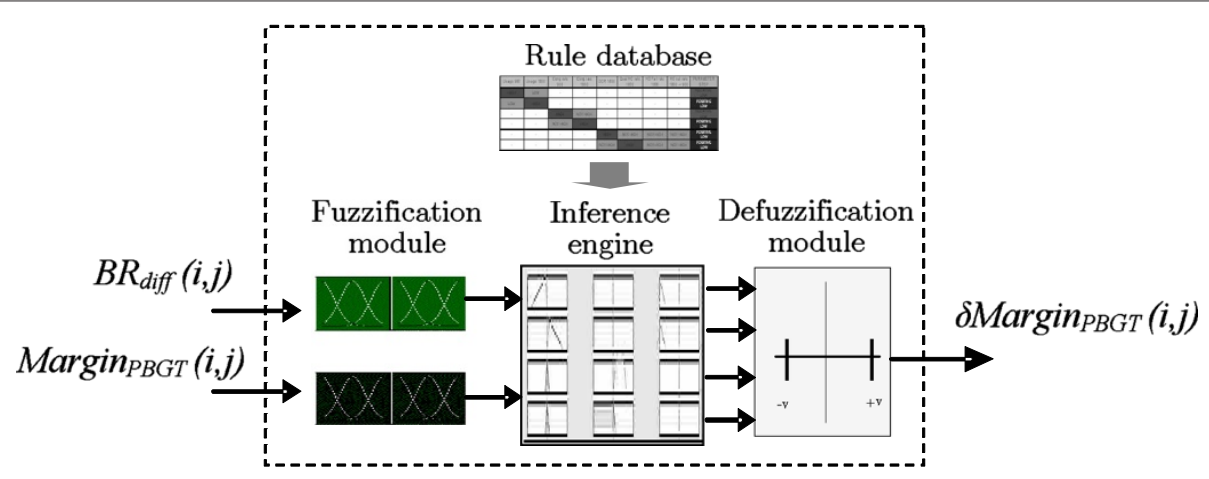

Figure 1 Structure of fuzzy logic controller for tuning margins. 


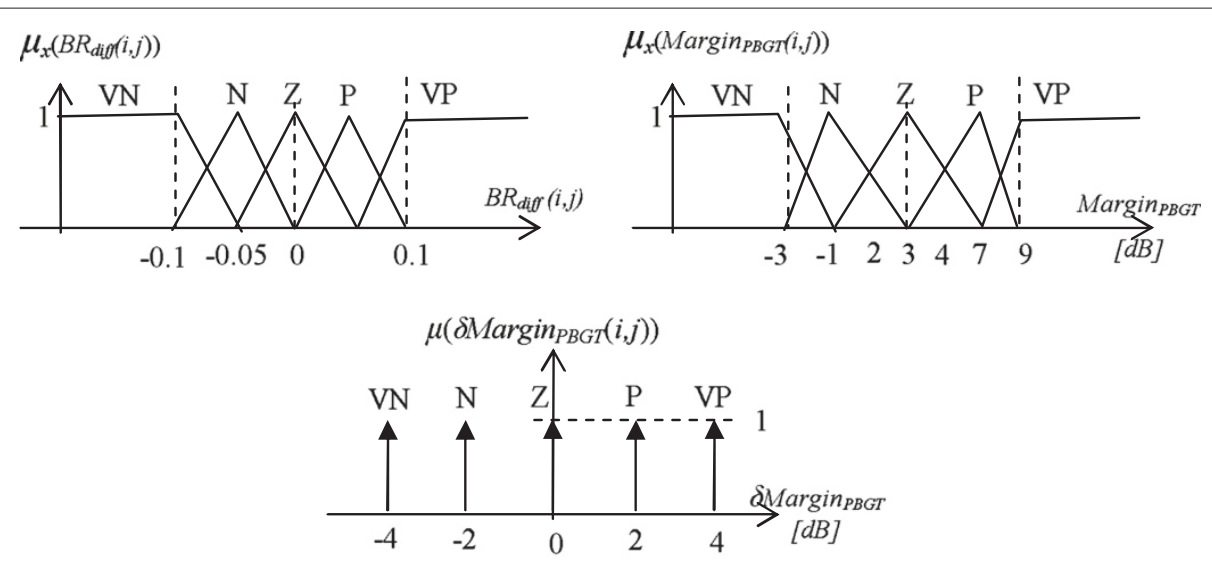

(a)

\begin{tabular}{ccc}
\hline$B R_{\text {diff }}(i, j)$ & $\operatorname{Margin}_{P B G T}(i, j)$ & SMargin $_{P B G T}(i, j)$ \\
\hline $\mathrm{VP}$ & - & $\mathrm{VN}$ \\
$\mathrm{P}$ & - & $\mathrm{N}$ \\
$\mathrm{N}$ & - & $\mathrm{P}$ \\
$\mathrm{VN}$ & - & $\mathrm{VP}$ \\
$\mathrm{Z}$ & $\mathrm{VN}$ & $\mathrm{P}$ \\
$\mathrm{Z}$ & $\mathrm{N}$ & $\mathrm{P}$ \\
$\mathrm{Z}$ & $\mathrm{P}$ & $\mathrm{N}$ \\
$\mathrm{Z}$ & $\mathrm{VP}$ & $\mathrm{N}$ \\
$\mathrm{Z}$ & $\mathrm{Z}$ & $\mathrm{Z}$ \\
\hline
\end{tabular}

(b)

Figure 2 Description of MTS fuzzy logic controller. (a) membership functions and (b) rules.

blocking difference, the more negative (positive) margin step. The last four rules implement a slow-return mechanism to restore the default margin value when no blocking is experienced just in case traffic steering is no longer necessary. In the defuzzification stage, the output value is obtained from the aggregation of all rules, for which the center-of-gravity method is adopted. This method calculates the output value as a weighted average. Weights are calculated from the degree of fulfilment of all rules, computed from their antecedents. For simplicity, all controllers are designed based on the Takagi-Sugeno approach, where output membership functions are constants, as shown in Figure 2a. The number of output membership functions has been selected large enough to allow fine parameter control.

As a result of tuning, very negative margin values could be reached. Such a negative value might cause that users are handed over to neighbor cells $j$ where $\overline{\operatorname{RSRP}}(j) \ll$ $\overline{\operatorname{RSRP}}(i)$, as deduced from (1). This might cause that the signal-to-noise and interference ratio (SINR) experienced by the handed-over user is significantly worse after the $\mathrm{HO}$ (note that the margin value is a rough approximation of the minimum SINR obtained by the user in the new cell). To avoid this problem, MTSC limits margin values by forcing that $\operatorname{Margin}_{\mathrm{PBGT}}(i, j) \geq-6.9$. This avoids that, after a $\mathrm{HO}$, SINR is below $-6.9 \mathrm{~dB}$ (i.e., the threshold below which the scheduler in the base station does not assign radio resources to a connection). The previous lower bound, when combined with (2), leads to the con-

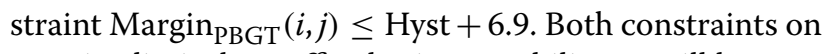
margins limit the traffic sharing capability, as will be seen in following section.

\section{Performance analysis}

As an alternative to modifying HO margins, PTS modifies transmit power to equalize traffic in a cell with its neighbors. Specifically, the transmit power of cell $i, P_{\mathrm{TX}}(i)$, is tuned through the addition of $\delta P_{\mathrm{TX}}(i)$ to reduce the difference in call blocking ratio against its neighbors. Since transmit power is a parameter defined on a cell basis, blocking differences must be defined accordingly. The inputs of the PTS FLC are the average blocking ratio difference, $\mathrm{BR}_{\text {diff }}(i)$, defined as

$$
\mathrm{BR}_{\mathrm{diff}}(i)=\mathrm{BR}(i)-\overline{\mathrm{BR}(N(i))}=\mathrm{BR}(i)-\frac{\sum_{j \in N(i)} \mathrm{BR}(j)}{|N(i)|},
$$


where $N(i)$ is the set of neighbors of cell $i$ and $|N(i)|$ is the number of neighbors of cell $i$, and the current deviation from the default (maximum) transmit power value, $\Delta P_{\mathrm{TX}}^{(n)}(i)$, defined as

$$
\Delta P_{\mathrm{TX}}^{(n)}(i)=P_{\mathrm{TX}}^{(n)}(i)-P_{\mathrm{TX}}^{(0)}(i),
$$

where superindex denotes time interval, and $P_{\mathrm{TX}}^{(n)}(i)$ and $P_{\mathrm{TX}}^{(0)}(i)$ are the current and initial (default, maximum) transmit power of cell $i$, respectively. Note the difference between $\Delta P_{\mathrm{TX}}^{(n)}$ (current power deviation from the default value) and $\delta P_{\mathrm{TX}}^{(n)}$ (power increment for this step). The latter is defined, similarly to (3), as

$$
P_{\mathrm{TX}}^{(n+1)}(i)=\operatorname{round}\left(P_{\mathrm{TX}}^{(n)}(i)+\delta P_{\mathrm{TX}}^{(n)}(i)\right) .
$$

Figure 3 depicts membership functions and rules for the PTS FLC. Roughly, the higher the blocking of the source cell compared to its adjacencies, the higher decrease in its transmit power. Again, note that $P_{\mathrm{TX}}(i)$ refers to both data and pilot transmit power.

In the previous section, different traffic sharing techniques for femtocell networks have been presented. In this section, several experiments are described to quantify the benefits and understand the limitations of the different approaches. For clarity, the analysis set-up is first introduced and results are then presented.

\section{Analysis set-up}

In the absence of an analytical model or live network trials to check the impact of strategies on network indicators, performance assessment is based on system-level simulations. The analysis set-up consists of the characterization of the simulation tool used in the tests and the methodology used to assess the methods.

\section{Simulation tool}

A three-dimensional enterprise scenario has been developed in a dynamic LTE system-level simulator [39]. Table 1 shows the properties of the simulator. The scenario includes an office building with femtocells in a larger scenario of $3 \times 2.6 \mathrm{~km}^{2}$ comprising a single macrocellular site consisting of three tri-sectorized cells. Thus, possible interaction between macrocells and femtocells is taken into account. Figure 4 shows the relative posi-
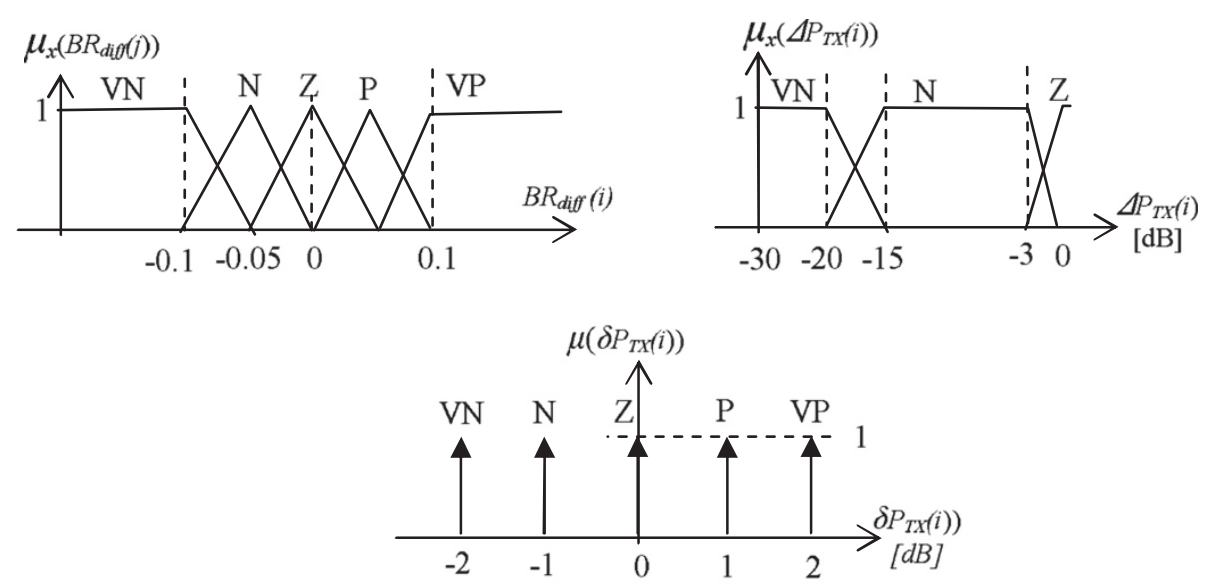

(a)

\begin{tabular}{ccc}
\hline$B R_{\text {dio }}(j)$ & $\operatorname{Margin}_{P X X}(i)$ & $\delta P_{T X}(i)$ \\
\hline VP & - & VN \\
P & - & $\mathrm{N}$ \\
$\mathrm{N}$ & $\mathrm{N}$ & $\mathrm{P}$ \\
$\mathrm{N}$ & $\mathrm{VN}$ & $\mathrm{P}$ \\
$\mathrm{Z}$ & $\mathrm{VN}$ & $\mathrm{P}$ \\
$\mathrm{Z}$ & $\mathrm{N}$ & $\mathrm{P}$ \\
$\mathrm{VN}$ & $\mathrm{N}$ & $\mathrm{P}$ \\
$\mathrm{VN}$ & $\mathrm{VN}$ & $\mathrm{VP}$ \\
$\mathrm{Z}$ & & $\mathrm{Z}$ \\
\hline
\end{tabular}

(b)

Figure 3 Description of PTS fuzzy logic controller. (a) membership functions and (b) rules. 
Table 1 Simulation parameters

\begin{tabular}{|c|c|c|}
\hline Time resolution & $100 \mathrm{~ms}$ & \\
\hline \multirow{4}{*}{$\begin{array}{l}\text { Propagation } \\
\text { model }\end{array}$} & indoor-indoor & Winner II A1 \\
\hline & indoor-outdoor & Winner II A2 \\
\hline & outdoor-outdoor & Winner II C2 \\
\hline & outdoor-indoor & Winner II C4 \\
\hline \multirow[t]{3}{*}{ BS model } & EIRP & 13 (femto)/43 (macro) dBm \\
\hline & Directivity & omni (femto)/tri-sector (macro) \\
\hline & Access & open access (macro/femto) \\
\hline \multirow[t]{2}{*}{ MS model } & Noise figure & $9 \mathrm{~dB}$ \\
\hline & Noise density & $-174 \mathrm{dBm} / \mathrm{Hz}$ \\
\hline \multirow[t]{2}{*}{ Traffic model } & Calls & Poisson (avg. 0.43 calls/user*h) \\
\hline & Duration & exponential (avg. 100 s) \\
\hline \multirow[t]{3}{*}{ Mobility model } & Outdoor & $3 \mathrm{~km} / \mathrm{h}$, random direction \& \\
\hline & & wrap-around \\
\hline & Indoor & random waypoint \\
\hline Service model & Voice over IP & $16 \mathrm{kbps}$ \\
\hline \multirow[t]{7}{*}{ RRM model } & $6 \mathrm{PRBs}(1.4 \mathrm{MHz})$ & \\
\hline & Cell Reselection & $\mathrm{C} 1-\mathrm{C} 2$ \\
\hline & Access control & Directed retry \\
\hline & & $\left(\mathrm{DR}_{\text {threshold }}=-44 \mathrm{dBm}\right)$ \\
\hline & Handover: & PBGT, Qual \\
\hline & Scheduler: RR-BC & Time: Round-Robin (RR) \\
\hline & & Freq.: best channel (BC) \\
\hline $\begin{array}{l}\text { Simulated } \\
\text { network time }\end{array}$ & $1 \mathrm{~h}$ (per loop) & \\
\hline
\end{tabular}

tion of macrocells and femtocells. The squares display the building and the area under study. The hexagons show cells included to avoid border effects by a wrap-around technique.

The distance between the macrocellular site and the building ( $500 \mathrm{~m}$ in this study) has been selected so that the signal level received from the macrocells inside the building is high enough to be considered as a source of interference, but low enough to avoid users inside the building connecting to the macrocell (which would make the analysis of traffic sharing between femtocells more difficult).

The considered propagation models are those of the Winner II project [41], considering indoor, outdoor, indoor-to-outdoor and outdoor-to-indoor environments. Shadowing is modeled by a spatially-correlated lognormal distribution with different standard deviation for indoor and outdoor users. Likewise, fast-fading is modeled by an Extended Indoor A (EIA) model for indoor users [42].
Several scenarios are considered in the tests, differing in the number of floors and femtocells per floor. In all of them, each floor can comprise up to four femtocells, whose location is pre-fixed. The floor plan and possible femtocell positions are the same in all floors. Figure 5 shows the layout of one of the floors. Dark circles represent femtocell positions, lines are walls, and small diamonds are working stations. A random waypoint mobility model is implemented for indoor users. Changes of floor are not considered. All adjacencies are considered for $\mathrm{HO}$ purposes inside the building.

Traffic demand inside the building is unevenly distributed both within floors and among floors, causing the need for traffic sharing among cells. To ease the analysis, the considered service model is Voice-over-IP.

The simulator includes common RRM features, such as CR, directed retry (DR), scheduling and PBGT and Quality HO. For simplicity, dropped calls are disabled in the simulations. For more details, the reader is referred to [39].

\section{Performance assessment methodology}

Assessment is carried out over three test scenarios of increasing complexity. The first two scenarios aim to show the capabilities and limitations of some of the techniques. A third scenario reflects an extreme, albeit more realistic, situation in which to quantify the benefits of the different approaches.

In each scenario, optimization techniques are tested along at least 25 optimization loops, each representing $1 \mathrm{~h}$ of network time. At the end of each loop, a controller collects performance statistics and changes network parameters, based on the membership functions and rules defined in Section 'Traffic steering algorithms'. Once parameters have changed, a new loop starts. The duration of each loop is long enough to ensure reliable performance statistics, while the large number of loops should ensure that the system reaches the steady state. The following paragraphs explain the experiments in each scenario.

1) Scenario 1, shown in Figure 6a, aims to show the ability of individual traffic steering approaches to balance the load within a floor. For this purpose, the scenario only comprises one floor with four femtocells located as in Figure 6a. All users are created in the service area of a femtocell (denoted as number 1), while no users are created in the other 3 femtocells in the same floor (number 2, 3, and 4). Such a distribution of traffic demand causes an extremely large blocking in femtocell 1 in the initial state (1st loop), when parameters are still in their default values. In this scenario, MTS and PTS are individually tested. The results will show the need for constraining MTS (i.e., MTSC). 

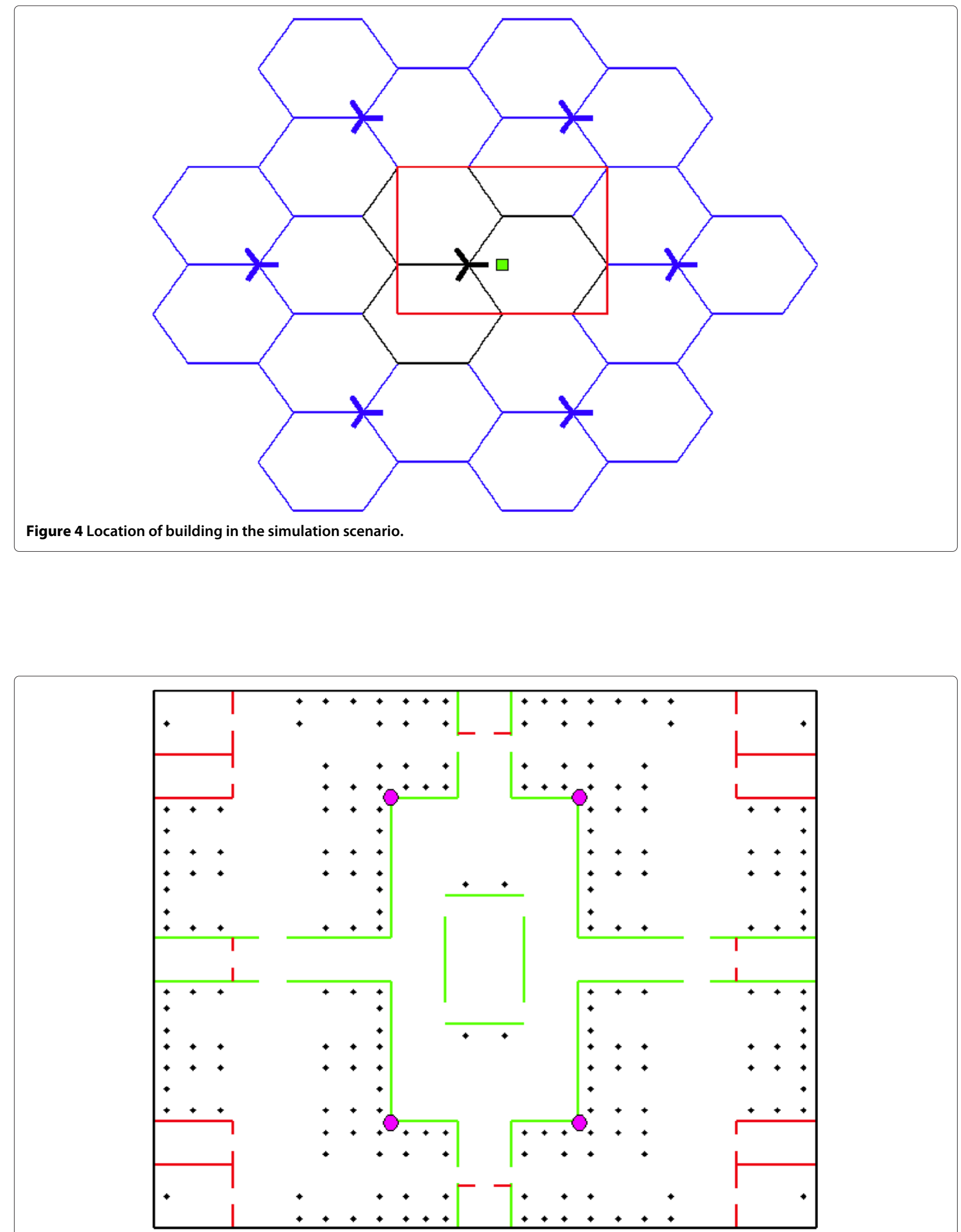

Figure 5 A floor diagram. 


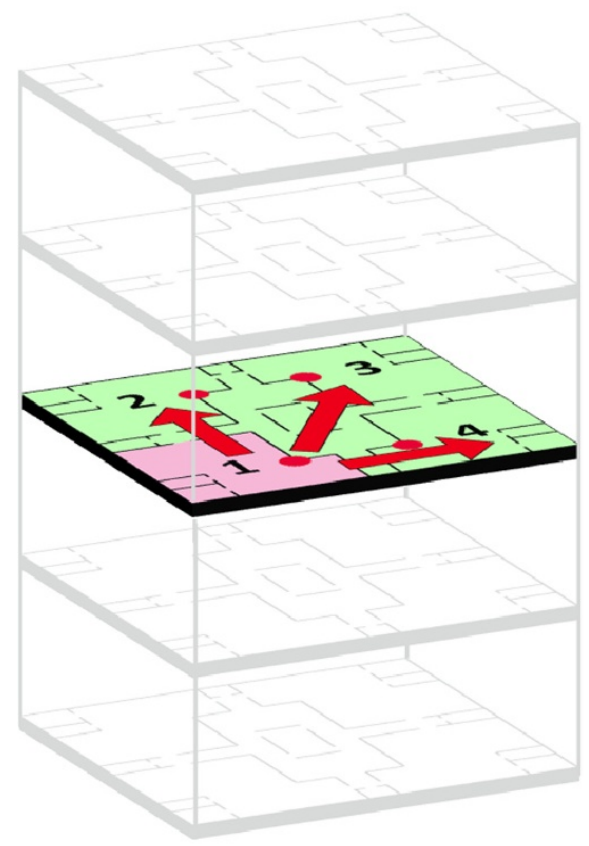

(a) Scenario 1

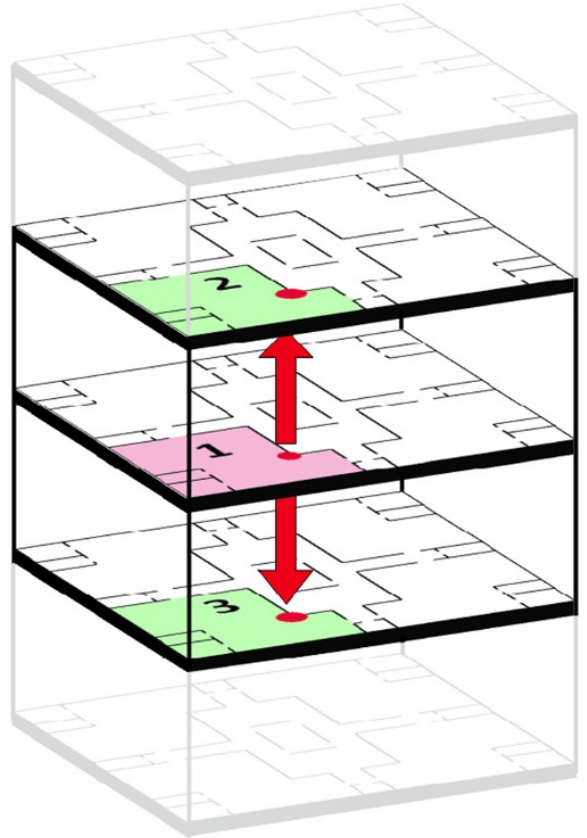

(b) Sccnario 2

Figure 6 Description of scenarios. (a) Scenario 1, and (b) Scenario 2.

It should be pointed that user movement is not restricted to the femtocell where it is created, but it can move to other rooms in the floor (but not to other floors) during the call. This has a negligible effect on traffic sharing.

2) Scenario 2, shown in Figure 6b, aims to highlight the limitations of some methods to balance traffic between cells of different floors. Consequently, this scenario comprises 3 floors, with only one active cell per floor. Cells in different floors are located in the same position in the floor plan. Traffic demand is distributed as in Scenario 1 (i.e., all users created in cell 1 , no users in cells 2 and 3 ), but propagation losses between cells are much larger due to the floor structure.

2) Scenario 3 is a generalization of Scenarios 1 and 2. It includes five floors and four femtocells per floor. A log-normal spatial traffic distribution is assumed, where the central floor is highly loaded with one of its cells experiencing extremely large call blocking, while upper and lower floors are underutilized. This can be considered as a worst-case situation, since most of the traffic is generated in a few cells, which are adjacent to each other. Different combinations of MTSC and PTS (including CTS) are tested, trying to overcome the limitations of individual techniques.

Several indicators are collected to rank the different network settings reached by the tuning methods. From the user perspective, performance measures are: a) the overall call blocking ratio (BR), defined as the ratio of blocked calls after DR against total attempts, as a measure of network capacity, and $b$ ) the outage ratio (OR), defined as the ratio of unserved connection time due to temporary lack of resources $\left(\mathrm{OR}_{r}\right)$ or bad SINR $\left(\mathrm{OR}_{q}\right)$, with $\mathrm{OR}=\mathrm{OR}_{r}+$ $\mathrm{OR}_{q}$, as a measure of network connection quality. For ease of analysis, BR and OR are aggregated into a single indicator, the unsatisfied user ratio (UUR), computed as $\mathrm{UUR}=\mathrm{BR}+\mathrm{OR}(1-\mathrm{BR})$. From the operator perspective, important measures are: c) the HO Ratio (HOR), defined by the ratio between the number of HOs and carried calls, as a measure of network signaling load, and d) the average deviation from the default value of margins and transmit power in cells and adjacencies of the scenario. Most of these statistics are available on a cell basis.

To find the best algorithm, the methodology described in [20] is used. The value of an algorithm is given by the performance of all network configurations reached during the optimization process. Note that, in self-tuning algorithms, such configurations are given by the series of parameter settings suggested by the controller as tuning progresses, which is referred to as a trajectory. In principle, the main focus of the analysis is on the asymptotic behavior, i.e., the value of $\mathrm{UUR}^{(n)}$ as $n \rightarrow \infty$. However, as self-tuning algorithms gradually change parameters in the real network, not only the final configuration (steady state) but also the whole trajectory (transient response) must be taken into account. 
To evaluate transient response by a single figure, an infinite-horizon discounted model [43] is considered, as in [20]. In this model, the overall penalty of a trajectory, $P$, is calculated as

$$
P=(1-\gamma) \sum_{n=0}^{\infty} \gamma^{n} \cdot \operatorname{UUR}^{(n)},
$$

(i.e., the weighted average of single penalties, UUR, across loops). This model takes into account long-term penalties, but future penalties are given less importance according to a geometric law with discount factor $\gamma$, where $0 \leq \gamma \leq 1$. The latter formula reflects that, in live environments, early rewards are preferred to delayed rewards, since traffic conditions might greatly vary with time and situations of persistent congestion are solved in the long term by other approaches. Strictly, an infinite number of loops should be simulated. To reduce the computational effort, it is assumed that equilibrium is reached after $h$ loops. Thus, the overall penalty is calculated as

$$
\begin{aligned}
P & =(1-\gamma) \sum_{n=0}^{h-1} \gamma^{n} \cdot \mathrm{UUR}^{(n)}+(1-\gamma) \sum_{n=h}^{\infty} \gamma^{n} \cdot \mathrm{UUR}^{(n)} \\
& \approx(1-\gamma) \sum_{n=0}^{h-1} \gamma^{n} \cdot \mathrm{UUR}^{(n)}+\gamma^{h} \cdot \mathrm{UUR}^{(h)}
\end{aligned}
$$

where $\mathrm{UUR}^{(h)}$ is the performance indicator value in the last simulated loop. Hereafter, $\gamma=0.95$ and $h=$ 24. Thus, 25 loops are simulated (i.e., 1 initial state + 24 tuning steps). The selected horizon should be large enough to ensure that the system has reached equilibrium. Even if this is not the case, the low value of $\gamma$ ensures that the influence of loops beyond this point is negligible.

\section{Simulation results}

\section{Scenario 1}

A preliminary analysis evaluates the sensitivity of cell dominance areas to parameter changes in the considered office scenario. For this purpose, the path-loss difference between a cell and the closest neighbor in every location is defined as

$$
\Delta L(x, i)=\min _{j}(L(x, j)-L(x, i)), \forall j \neq i
$$

where $L(x, i)$ is the pathloss from base station $i$ to position $x$ in $\mathrm{dB}$. The min operation ensures that $\Delta L$ always shows the difference with the closest neighbor in that location. Figure 7 shows the referred difference for cell 1 (i.e., $\Delta L(x, 1)$ in Scenario 1$)$. In the figure, cell 1 is located in the left lower part. For clarity, contour lines are $4 \mathrm{~dB}$ apart. A positive value indicates that users in that position will be attached to cell 1 if parameters are set to default values (i.e., maximum transmit power and positive $\mathrm{HO}$ margins). Likewise, $\Delta L$ quantifies the deviation of $\mathrm{HO}$ margin or transmit power needed to send users from cell 1 to a neighbor cell or viceversa. From the figure, it can be deduced, for instance, that most users in corridors could be re-assigned to cell 1 by ensuring that $P_{\mathrm{TX}}(j)-P_{\mathrm{TX}}(1)+\operatorname{Margin}_{\mathrm{PBGT}}(j, 1) \leq-12 \mathrm{~dB}$, either by
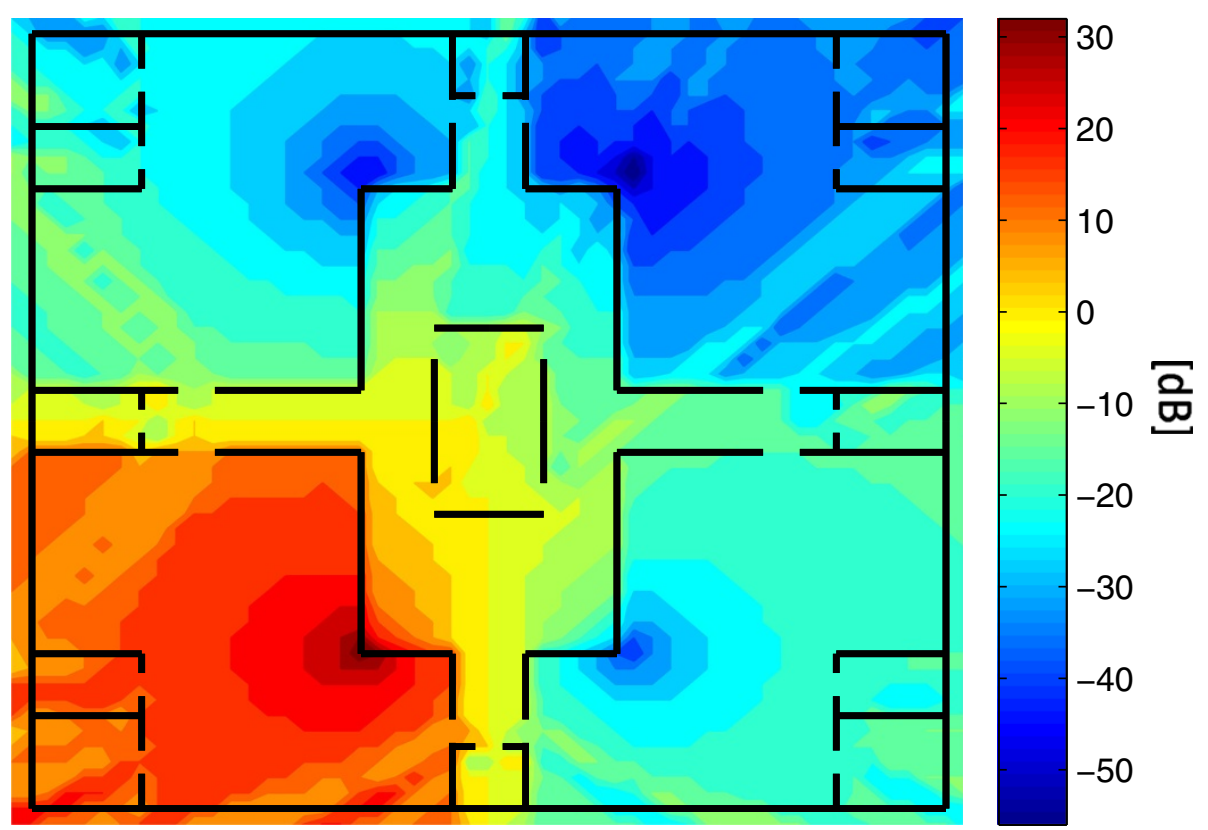

Figure 7 Path loss differences between cells in Scenario 1. 
displacing margins up to $-12 \mathrm{~dB}$ or by reducing transmit power of neighbor cells by $12 \mathrm{~dB}$. Similarly, half of the users in cell 1 could be handed over to surrounding cells by forcing that $P_{\mathrm{TX}}(j)-P_{\mathrm{TX}}(1)-\operatorname{Margin}_{\mathrm{PBGT}}(1, j) \geq 12 \mathrm{~dB}$. In contrast, to send users in the top-right area to cell 1 , extreme changes of margins or transmit power would be necessary to compensate for the multiple wall losses. From the figure, it is also envisaged that smooth traffic steering is hard to achieve by tuning parameters in this scenario. Note that, due to small propagation differences within a room, once the wall losses are overcome, all the users in a room (and not only a few of them, as would be desirable) are re-allocated to the same cell.

In this scenario, MTS decreases the HO margins of cell 1 to send users to cells 2, 3, and 4. Figures 8 and 9 show the evolution of average outgoing $\mathrm{HO}$ margin in cell 1 (i.e., mean $\left.\left(\operatorname{Margin}_{\mathrm{PBGT}}(1, j)\right)\right)$ and UUR, respectively, after 20 loops in MTS. To aid the analysis, UUR figures are broken down in $\mathrm{OR}_{r}, \mathrm{OR}_{q}$ and $\mathrm{BR}$ contributions. In Figure 9, it is observed that, in the first three loops, UUR decreases sharply. As expected, the MTS controller decreases outgoing margins in cell 1 to equalize blocking rates in the floor. The more negative margin values, the larger the service area of cell 1 that is re-converted into service area of other cells. Traffic sharing is first carried out by re-allocating users next to corridors or doors in cell 1 , as can be deduced from Figure 7. Thus, MTS needs to change HO margins by $8 \mathrm{~dB}$ (i.e. from 3 to $-5 \mathrm{~dB}$ ) to decrease UUR from 16 to $12 \%$ in the first three loops. Thereafter, since balance has not been reached, MTS keeps decreasing outgoing margins trying to send more users out of cell 1 . As a result, margins becomes very negative (i.e., below $-6.9 \mathrm{~dB}$ ). In this situation, users close to the congested femtocell are sent to neighbor cells, experiencing high interference from

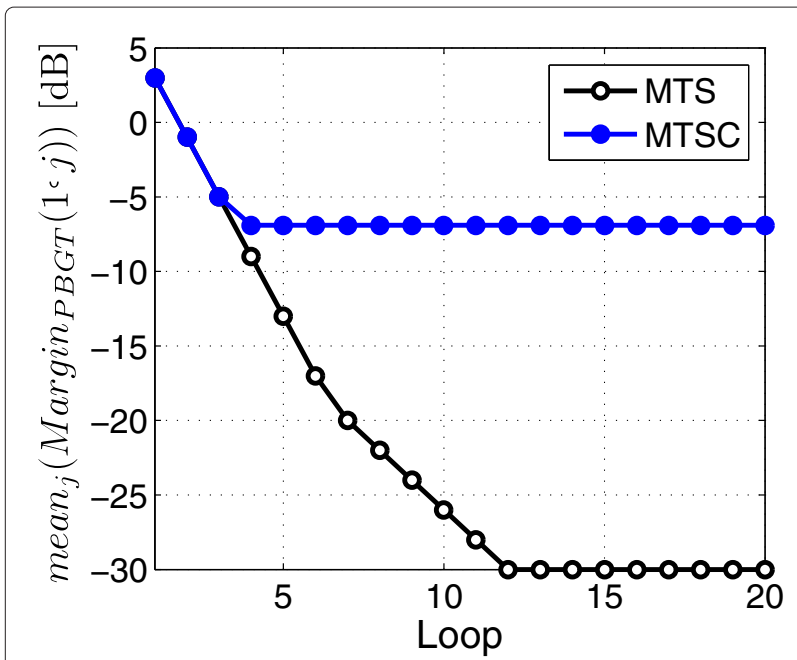

Figure 8 Margin deviations for MTS and MTSC in Scenario 1.

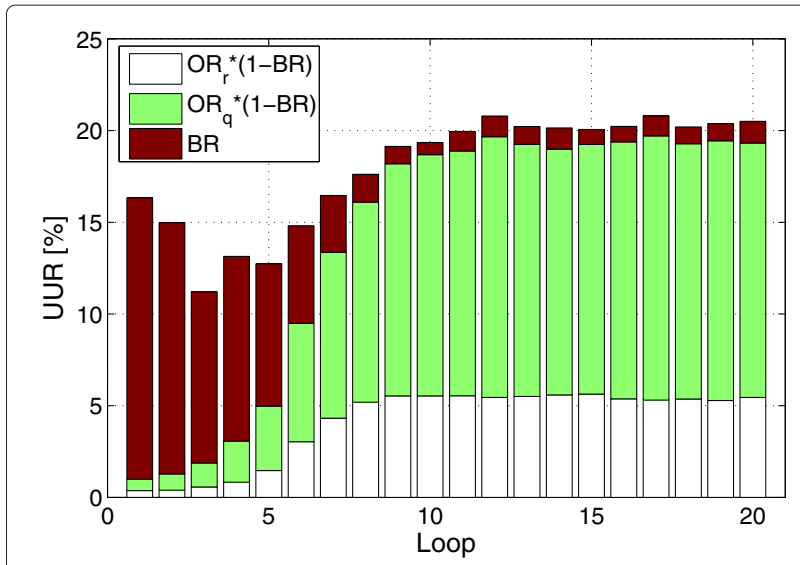

Figure 9 MTS performance in Scenario 1.

the original cell. Thus, $\mathrm{OR}_{q}$ increases significantly. As a consequence, even if MTS manages to decrease BR in the first three loops, it ends up with an UUR worse than in the initial state. Not shown is the fact that HOR also increases from 1 to 13 (i.e., from 1 to $13 \mathrm{HOs}$ per call). This value clearly indicates a ping-pong effect when margins are excessively low. Such an impairment can be avoided by forcing that $\mathrm{HO}$ margins are always above $-6.9 \mathrm{~dB}$, justifying the use of limits in Margin ${ }_{\mathrm{PBGT}}$ (which is the only difference of MTSC compared to MTS). Of course, this is achieved at the expense of limiting the traffic sharing capability. Nonetheless, in the $3^{r d}$ loop, when MTS has not reached its limits, UUR is already $4 \%$ lower in absolute terms than in the initial state. Note that, due to (2), a lower bound of $-6.9 \mathrm{~dB}$ in Margin PBGT $_{\text {imposes an upper }}$ bound of $12.9(=6-(-6.9)) \mathrm{dB}$. Obviously, MTSC and MTS perform the same for Margin ${ }_{\mathrm{PBGT}}$ values inside the allowed interval (i.e., $[-6.912 .9] \mathrm{dB}$ ).

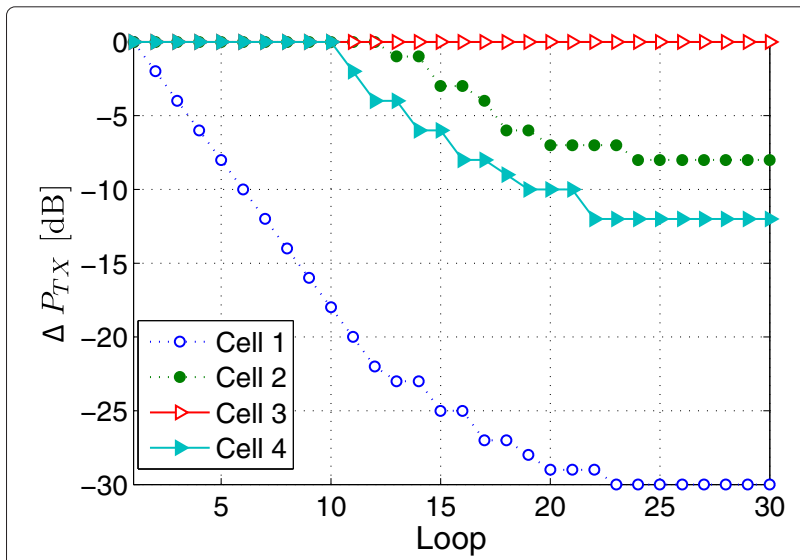

Figure 10 Transmit power deviations for PTS in Scenario 1. 
PTS shows a similar behavior in Scenario 1. Figure 10 presents the power deviation in the scenario for each cell. Figure 11 presents the UUR obtained by PTS. In the first five loops, power decrements in cell 1 are directly translated into UUR reduction. In the next 5 loops (loop 6 to 10 ), cell 1 still tries to send more users to other cells, but OR increases while UUR keeps the same. Basically, by sending users from cell 1 to other cells, BR decreases, but OR increases. From loop 10, cells 2 and 4 experience blocking and start to decrease their power, trying to send users to cell 3, which is the more distant cell. In contrast to MTS, there is no need for limiting power deviations, since UUR does not degrade with large values of $\Delta P_{\mathrm{TX}}$. Likewise, there is no ping-pong effect in PTS, because changes in pilot power causes that users start their calls in the cell providing the strongest signal level, making HOs unnecessary.

\section{Scenario 2}

Scenario 2 contains three cells in different floors. Propagation losses due to floors are $17 \mathrm{~dB}$, whereas those of light walls between rooms are $5 \mathrm{~dB}$. Hence, traffic sharing techniques in Scenario 2 need larger parameter changes than in Scenario 1. Due to above-mentioned constraints, MTSC can not steer traffic in Scenario 2. A closer analysis shows that users start to be handed over from cell 1 to cells 2 and 3 in the upper/lower floor when margins are below $-17 \mathrm{~dB}$. Such a value is beyond MTSC constraints. Should constraints be disabled (as in MTS), UUR would degrade as in Scenario 1.

In contrast, PTS manages to overcome floor losses. Figure 12 illustrates the evolution of UUR with PTS in the new scenario. In the figure, it is observed that network performance stays the same in the first five loops, even if $\Delta P_{\mathrm{TX}}(1)$ has changed. Hitherto, the power decrease in cell 1 is not enough to compensate for floor attenuation. Thereafter, reducing the transmit power of cell 1 causes that users in the middle floor start to be attached

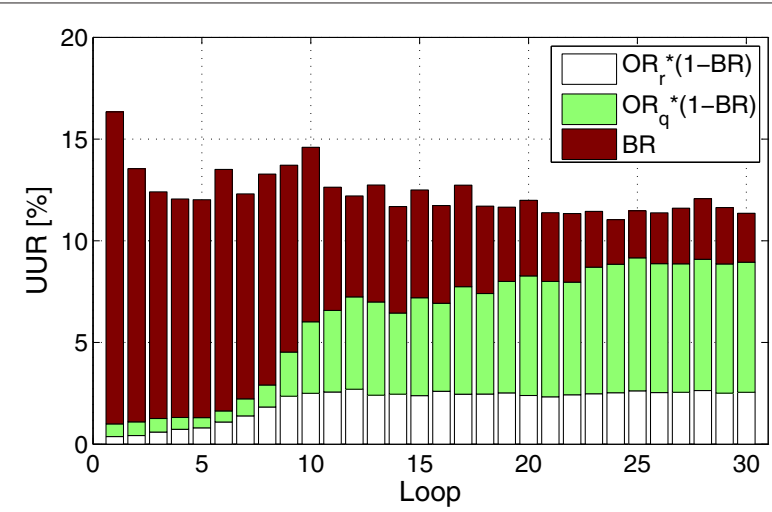

Figure 11 PTS performance in Scenario 1. to cells in other floors, and BR decreases. However, OR also increases, partly due to the impairment of connection quality to users in cell 1 . As a result, UUR keeps the same. Thus, PTS does not achieve any overall gain in this scenario because of the excessive isolation between cells of different floors.

It should be pointed out that UUR values in Figure 12 are much higher than in Figure 11 (i.e., 40\% versus 16\% for the first loop). This is due to the fact that, in Scenario 1, neighbor cells are in the same floor, so users created in cell 1 can be handed over easier to other cells in the same floor. In Scenario 2, neighbor cells experience more attenuation so that users created in cell 1 receive a very low signal level, making HOs difficult and increasing the UUR.

\section{Scenario 3}

Previous scenarios have been used to check the limitations of simple traffic sharing algorithms. Scenario 3 considers a more realistic scenario with several cells per floor. The analysis is now focused on the combination of techniques to overcome the limitations of individual approaches. Such combined strategies are:

1) MTSC before PTS (MTSC-PTS). MTSC is enabled only for the first 25 loops and PTS is then activated for the rest of the simulation. It is expected that MTSC reaches equilibrium before loop 25. Thus, the effects of MTSC in Scenario 3 can be observed in the first 25 loops, and later improvements achieved by PTS can be analyzed in the last 25 loops. Note that, in this technique, PTS is enabled when margins are not in their default values.

2) PTS before MTSC (PTS-MTSC). Similar to the previous strategy, but methods are enabled in different order, i.e., PTS is activated in the first half of the simulation and MTSC in the second half. Thus, the effect of PTS is evaluated first, and MTSC

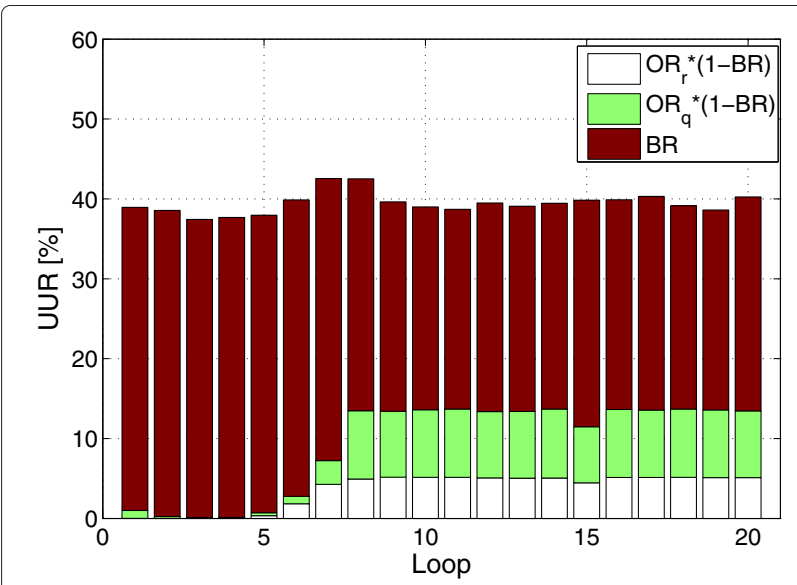

Figure 12 PTS performance in Scenario 2. 
improvements are checked later. In this case, MTSC is enabled when power values have already changed.

3) Uncoordinated MTSC and PTS (MTSC\&PTS). Both MTSC and PTS are executed simultaneously in all the 50 loops. Such an strategy aims to check if there is any problem or benefit in running both algorithms in parallel.

4) Coordinated Traffic Sharing (CTS). This strategy has been explained in Section 'Traffic steering algorithms'. Unlike MTSC-PTS and PTS-MTSC, in CTS, switching between strategies does not take place in pre-fixed instants, but these are defined during execution on a cell basis. In each cell, MTSC is activated first. Then, switching to PTS occurs when MTSC cannot improve network performance. Thus, PTS is enabled in cell $i$ when, in all its adjacencies, either MTSC has reached its limits (because margins have reached their upper or lower limits) or traffic balance has been reached. These conditions can be expressed as follows:

$$
\begin{aligned}
& \operatorname{Margin}_{\mathrm{PBGT}}(i, j) \leq-6.9\left|\operatorname{Margin}_{\mathrm{PBGT}}(i, j) \geq 12.9\right| \\
& \mathrm{BR}_{\text {diff }}(i, j)<0.02 \forall j \in N(i) .
\end{aligned}
$$

Note that, to switch from MTSC to PTS, at least one of the three conditions must be satisfied in every adjacent cell $i$. Obviously, the first and second terms in (10) cannot be fulfilled at the same time for the same adjacency. However, different adjacencies can satisfy different conditions.

Figure 13a-e show the evolution of UUR, BR, OR, HOR, and $\overline{\Delta P_{\mathrm{TX}}}$ for all strategies, respectively. In Figure 13a, it is clear that all combined strategies decrease UUR similarly, reaching values around $8 \%$ in equilibrium. Such a result is due to a reduction of BR, as observed in Figure 13b, and is achieved at the expense of deteriorating the overall connection quality, as deduced from Figure 13c.

Compared to non-combined strategies, MTSC-PTS clearly outperforms MTSC figures (i.e., UUR $\approx 12 \%$ for $n=25$ and decreases up to $8 \%$ for $n=50$ after PTS). However, PTS-MTSC, achieves no gain in UUR compared to the basic PTS approach. The same is true for MTSC\&PTS and CTS strategies, whose only difference is that they reach equilibrium faster. The latter was expected since they modify both margins and transmit powers at the same time.

All methods achieve the traffic sharing effect by reallocating users in the congested cell to a different cell. When this action is performed by the HO process (as in MTSC), the number of HOs is significantly increased, which is observed in Figure 13d. Such an increase is

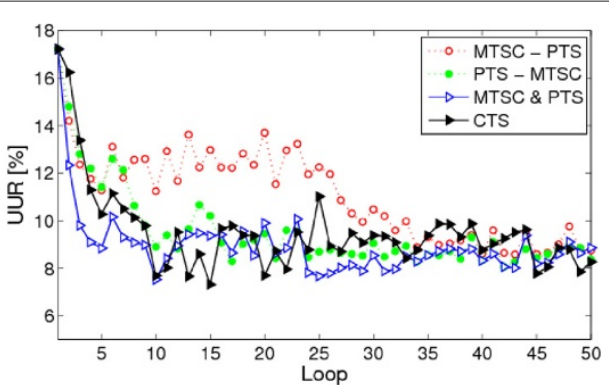

(a) Unsatisfied users ratio

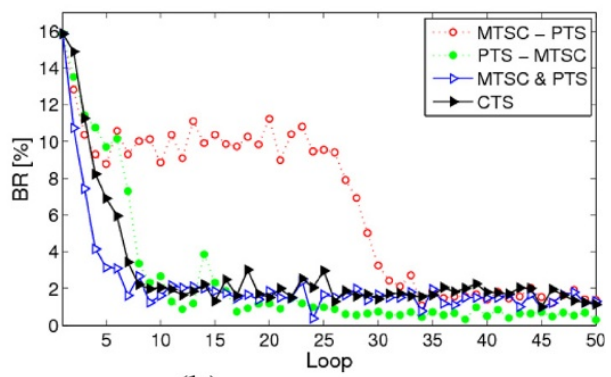

(b) Blocking ratio

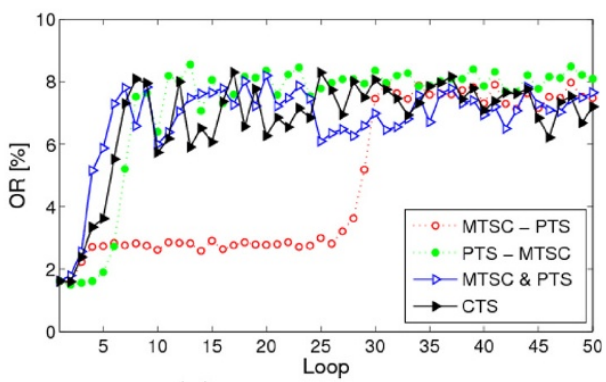

(c) Outage ratio

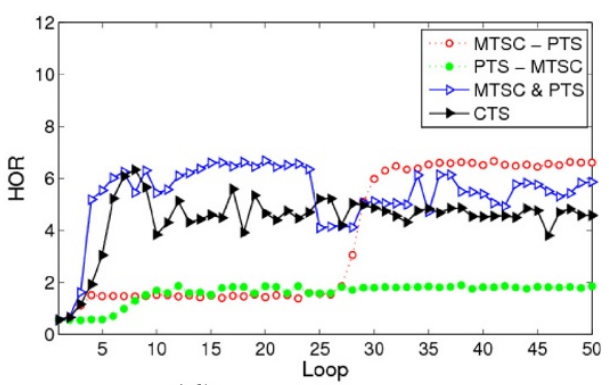

(d) Handover ratio

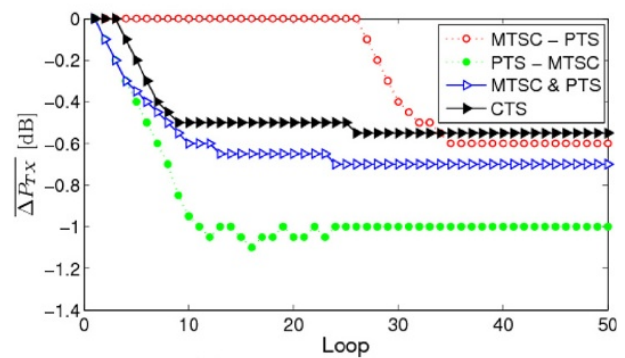

(e) Global average power deviation

Figure 13 Performance of combined techniques in Scenario 3. 
extremely large when ping-pong HOs are generated from users experiencing low SINR in the target cell, returning back to the original (congested) cell and being sent back again to the target cell. This is the case of MTSC\&PTS and CTS, where a six-fold increase is obtained. In contrast, in the first stage of MTSC-PTS and PTS-MTSC HOR is low.

Although all methods have similar asymptotic behavior for performance indicators, their trajectories are quite different. Table 2 shows the overall penalty, $P$, together with the minimum and final UUR values, for the trajectory of the different methods. Although all strategies reach a similar equilibrium state (4th column in Table 2), CTS, and especially MTSC\&PTS, reach the equilibrium before than others and their $P$ values are lower than fixed-transition strategies (i.e., MTSC-PTS and PTS-MTSC).

From the results presented so far, it might be deduced that MTSC\&PTS is the best strategy, as it achieves the best UUR faster. However, when power deviations are taken into account, the conclusion is not the same. Table 2 shows that the trajectory of CTS is quite similar to that of MTSC\&PTS, but Figure 13e presenting the average power deviation in the scenario, $\triangle \mathrm{PTX}$, i.e.,

$$
{\overline{\Delta P_{T X}}}^{(n)}=\frac{\sum_{i=1}^{N_{\text {cells }}} \Delta P_{T X}^{(n)}(i)}{N_{\text {cells }}},
$$

where $N_{\text {cells }}$ is the number of cells in the scenario, shows that the same network performance is reached with less average power deviation.

A closer analysis (not shown here) reveals that the largest transmit power deviation, $\max _{i}\left(\Delta P_{\mathrm{TX}}^{(50)}(i)\right)$ (i.e., the maximum transmit power for any cell at the end of the tuning process) in MTSC-PTS, PTS-MTSC, MTSC\&PTS and CTS are $-10,-16,-11$, and $-9 \mathrm{~dB}$, respectively.

From these results, it can be concluded that there is no method that outperforms all others in all indicators. All techniques reach a similar value of UUR (8\%) at the end of the optimization process. CTS and MTSC\&PTS are the best methods in terms of adaptation speed, since they reach the steady state faster, as deduced from their lower $P$ value. If power deviations have to be minimized, CTS is the best method, since it leads to the smallest average and maximum power deviations $(-0.5$ and $-9 \mathrm{~dB}$, respectively). If power modification is not an issue,

\section{Table 2 Penalty values for combined strategies}

\begin{tabular}{lccc}
\hline Strategy & $\boldsymbol{P}$ & $\boldsymbol{m i n}\left(\boldsymbol{U U R}^{(\boldsymbol{n})}\right)$ & $\boldsymbol{U U \boldsymbol { R }}(\mathbf{5 0})$ \\
\hline MTSC-PTS & 0.1187 & 0.0858 & 0.0876 \\
PTS-MTSC & 0.1040 & 0.0806 & 0.0836 \\
MTSC\&PTS & 0.0947 & 0.0752 & 0.0886 \\
CTS & 0.1016 & 0.0732 & 0.08266 \\
\hline
\end{tabular}

PTS-MTSC is the preferred option, since its HOR is less than half that of CTS. Nonetheless, note that HOR can be reduced by other means (e.g., tuning cell reselection parameters [20]).

All the results presented here have been obtained in a regular scenario, where femtocells are located symmetrically in a floor and similarly in all floors. A comprehensive sensitivity analysis has shown that the regular scenario is a worst-case scenario for MTS and PTS, i.e., the improvement in UUR obtained with them in any irregular scenario is larger than in the regular scenario. By contrast, the regular scenario proves to be an average case for MTSC and all combined techniques (i.e., MTSC-PTS, PTS-MTSC, MTSC\&PTS, and CTS). More importantly, all methods (except MTS) manage to improve the UUR in all irregular scenarios, which is a strong evidence of the robustness of their traffic sharing approach.

\section{Conclusions}

In this study, several methods have been proposed for traffic sharing in an enterprise LTE femtocell scenario. The methods are based on tuning handover margins and/or femtocell transmit power by fuzzy logic controllers. Simulation results in several scenarios have shown that the proposed methods can decrease call blocking, but some of them deteriorate network connection quality significantly. Having identified interference from the originally congested femtocell as an important limitation, the variation of handover margins has been restricted. Thus, part of the congestion relief effect is achieved while connection quality is kept almost unaltered. Once limitations of simple methods have been characterized, combined strategies have been designed to overcome the limitations of individual approaches. Results have shown that, by making the most of $\mathrm{HO}$ margins first, deviation of transmit powers can be kept to a minimum.

The algorithms proposed in this study intend to solve persistent congestion problems by slowly changing parameters based on statistical indicators. To obtain reliable statistics, the measurement period must be large enough (e.g., $1 \mathrm{~h}$ ), which limits the frequency of parameter changes and hence the capability to cope with fast traffic fluctuations. However, the methods can easily be adapted to cope with daily traffic fluctuations by defining time slots of several hours and tuning parameters based on measurements of the same period in the previous day [11].

All the tested methods could be run in a centralized node or in a distributed manner as long as statistics of neighbors are available in femtocells. Such piece of information can be provided by the central node since parameters are modified slowly. Likewise, the methods can also be applied to other scenarios with open femtocells (e.g., airports). 


\section{Abbreviations}

BR: Blocking ratio; CR: Cell reselection; CTS: Combined traffic sharing; DR: Directed retry; EIA: Extended indoor A; FLC: Fuzzy logic controller; HO: HandOver; HOR: HO ratiol; LTE: Long term evolution; MTS: Margin traffic sharing; MTSC: Constrained MTS; OFDMA: Orthogonal frequency division multiple access; OR: Outage ratio; PBGT: Power BudGeT; PTS: Power traffic sharing; RRM: Radio resource management; SINR: Signal to noise and interference ratio; SON: Self organizing network; UMTS: Universal mobile telecommunications system; UUR: Unsatisfied user ratio; WiMAX: Wireless interoperability for microwave access.

\section{Competing interests}

The authors declare that they have no competing interests.

\section{Acknowledgements}

This study had been funded by the Spanish Ministry of Science and Innovation (grant TEC2009-13413) and Junta de Andalucía (grant TIC-4052).

Received: 21 November 2011 Accepted: 1 October 2012

Published: 12 November 2012

\section{References}

1. S Saunders, in 2nd Intl. Conference on Home Access Points and Femtocells. The role of cooperation in establishing an efficient femto economy, (London, 2007), pp. 1-5

2. J Cullen, in Femtocell Europe. Radioframe presentation (London, 2008)

3. H Zhang, X Wen, B Wang, W Zheng, Y Sun, in Second International Conference on Communication Software and Networks, 2010. ICCSN'10. A novel handover mechanism between femtocell and macrocell for LTE based networks, (Singapore, 2010), pp. 228-231

4. V Chandrasekhar, J Andrews, A Gatherer, Femtocell networks: a survey, IEEE Commun. Mag. 46(9), 59-67 (2008)

5. J Ramiro, K Hamied, Self-Organizing Networks: Self-Planning, Self-Optimization and Self-Healing for GSM, UMTS and LTE (John Wiley \& Sons, New York, 2011)

6. G Americas, The benefits of SON in LTE: Self-optimizing and self-organizing networks, Tech. rep (2009)

7. Next Generation Mobile Networks Recommendation on SON and O\&M Requirements, Tech. rep. NGMN (2008)

8. J Kojima, K Mizoe, Radio mobile communication system wherein probability of loss of calls is reduced without a surplus of base station equipment. U.S Patent 4435840 (1984)

9. C Saraydar, A Yener, Adaptive cell sectorization for CDMA systems, IEEE J. Sel. Areas Commun. 19(6), 1041-1051 (2001)

10. N Papaoulakis, D Nikitopoulos, S Kyriazakos, in 12th IST Mobile and Wireless Communications Summit. Practical radio resource management techniques for increased mobile network performance (Aveiro, 2003)

11. VWille, S Pedraza, M Toril, R Ferrer, J Escobar, Trial results from adaptive hand-over boundary modification in GERAN, Electron. Lett. 39(4), 405-407 (2003)

12. S Kourtis, R Tafazolli, in Proc. 51st IEEE Vehicular Technology Conference (VTC). Adaptive handover boundaries: a proposed scheme for enhanced system performance, vol 3, (Tokio, 2000), pp. 2344-2349

13. R Nasri, Z Altman, H Dubreil, Z Nouir, in Proc. 63rd IEEE Vehicular Technology Conference (VTC). WCDMA downlink load sharing with dynamic control of soft handover parameters, vol 2, (Melbourne, 2006), pp. 942-946

14. R Nasri, Z Altman, in 5th Int. Conf. on Advances in Mobile Computing and Multimedia. Handover adaptation for dynamic load balancing in 3GPP long term evolution systems, vol 230, (Jakarta, 2007), pp. 145-154

15. A Lobinger, S Stefanski, T Jansen, I Balan, in Proc. IEEE 71st Vehicular Technology Conference (VTC). Load balancing in downlink LTE self-optimizing networks, (Taipei, 2010), pp. 1-5

16. R Kwan, R Arnott, R Paterson, R Trivisonno, M Kubota, in Proc. IEEE 72nd Vehicular Technology Conference (VTC). On mobility load balancing for LTE systems, (Ottawa, 2010), pp. 1-5

17. P Muñoz, R Barco, I De la Bandera, M Toril, S Luna-Ramírez, in Proc. IEEE 73rd IEEE Vehicular Technology Conference (VTC). Optimization of a fuzzy logic controller for handover-based load balancing, (Budapest, 2011), pp. 1-5

18. TChandra, W Jeanes, H Leung, in Proc. 47th IEEE Vehicular Technology Conference (VTC). Determination of optimal handover boundaries in a cellular network based on traffic distribution analysis of mobile measurement reports, vol 1, (Phoenix, 1997), pp. 305-309

19. J Steuer, K Jobmann. The use of mobile positioning supported traffic density measurements to assist load balancing methods based on adaptive cell sizing, vol 1, (Lisboa, 2002), pp. 339-343

20. M Toril, $V$ Wille, Optimization of handover parameters for traffic sharing in GERAN, Wirel. Personal Commun. 47(3), 315-336 (2008)

21. Z Altman, C Balageas, P Beltran, Ezra Ben Y, E Formet, J Hamalainen, O Marcé, E Mutafungwa, S Perales, M Ran, Z Zheng, in Proc. 21st IEEE Int. Symp. on Personal, Indoor and Mobile Radio Communications (PIMRC) Workshops. Femtocells: the HOMESNET vision, (Instanbul, 2010), pp. 139-144

22. http://www.ict-befemto.eu

23. G Vivier, M Kamoun, Z Becvar, E de Marinis, Y Lostanlen, A Widiawan, in Future Network \& Mobile Summit. Femtocells for next-G wireless systems: the FREEDOM approach, (Florence, 2010), pp. 1-9

24. H Claussen, L Ho, L Samuel, An overview of the femtocell concept, Bell Labs Tech. J. 13, 221-245 (2008)

25. H Claussen, L Ho, L Samuel, in Wireless Telecommunications Symposium (WTS). Self-optimization of coverage for femtocell deployments, (Pomona, 2008), pp. 278-285

26. H Claussen, F Pivit, in IEEE International Conference on Communications (ICC). Femtocell coverage optimization using switched multi-element antennas, (Dresden, 2009), pp. 1-6

27. Z Becvar, P Mach, in Proc. 6th International Conference on Wireless and Mobile Communications (ICWMC). Adaptive hysteresis margin for handover in femtocell networks, (Valencia, 2010), pp. 256-261

28. A Tyrrell, F Zdarsky, E Mino, M Lopez, in Proc. 73rd IEEE Vehicular Technology Conference (VTC). Use cases, enablers and requirements for evolved femtocells, (Budapest, 2011), pp. 1-5

29. D Lopez-Perez, A Valcarce, G de la Roche, J Zhang, O F D M A femtocells: A roadmap on interference avoidance, IEEE Commun. Mag. 47(9), 41-48 (2009)

30. D López-Pérez, X Chu, A Vasilakos, in Special Interest Group on Data Communications (SIGCOMM). H Claussen, Minimising cell transmit power: towards self-organized resource allocation in OFDMA femtocells, (Toronto, 2011), pp. 410-411

31. A Galindo, L Giupponi, M Dohler, in Proc. IEEE Global Communications Conference (GLOBECOM). Cognition and docition in OFDMA-based femtocell networks, (Miami, 2010), pp. 1-6

32. S Namal, K Ghaboosi, M Bennis, A MacKenzie, M Latva-aho, in 44th Conf. on Signals, Systems and Computers (ASILOMAR). Joint admission control and interference avoidance in self-organized femtocells, (Pacific Groove, 2010), pp. 1067-1071

33. M Bennis, L Giupponi, E Diaz, M Lalam, M Maqbool, E Strinati, A De Domenico, M Latva-aho, in 2nd International Conference on Wireless Communication, Vehicular Technology, Information Theory and Aerospace Electronic Systems Technology (Wireless VITAE). Interference management in self-organized femtocell networks: the BeFEMTO approach, (Chennai, 2011), pp. 1-6

34. S Barbarossa, S Sardellitti, A Carfagna, P Vecchiarelli, in Proc. Int. Conf. on Cognitive Radio Oriented Wireless Networks and Communications (CrownCom). Decentralized interference management in femtocells: a game-theoretic approach, (Cannes, 2010), pp. 1-5

35. I Ashraf, H Claussen, L Ho, in Proc. IEEE International Conference on Communications (ICC). Distributed radio coverage optimization in enterprise femtocell networks, (Cape Town, 2010), pp. 1-6

36. K Han, Y Choi, D Kim, M Na, S Choi, K Han, in 7th Int. Symp. on Modeling and Optimization in Mobile, Ad Hoc, and Wireless Networks, (WiOPT). Optimization of femtocell network configuration under interference constraints, (Seoul, 2009), pp. 1-7

37. M Simsek, T Akbudak, B Zhao, A Czylwik, in International ITG Workshop onSmart Antennas (WSA). An LTE-femtocell dynamic system level simulator, (Bremen, 2010), pp. 66-71

38. D Rose, T Jansen, T Kurner, in Proc. 73rd IEEE Vehicular Technology Conference (VTC). Modeling of femto cells - simulation of interference and handovers in LTE networks, (Budapest, 2011), pp. 1-5

39. J Ruiz-Avilés, Ramírez Luna S, M Toril, F Ruiz, I de la Bandera Cascales, P Muñoz Luengo, in Proc. 4th International Workshop on Femtocells, Wireless Advanced (WiAd). Analysis of load sharing techniques in enterprise LTE femtocells, (London, 2011), pp. 195-200 
40. T Ross, Fuzzy Logic with Engineering Applications. (McGraw-Hill, New York, 1995)

41. D1.1.2. WINNER II Channel Models. Part II. Radio Channel Measurement and Analysis Results. v1.0 Tech. rep. WINNER II IST project (2007)

42. T Sorensen, P Mogensen, F Frederiksen, in IEEE 62nd Vehicular Technology Conference (VTC). Extension of the ITU channel models for wideband (OFDM) systems, vol 1, (Dallas, 2005), pp. 392-396

43. L Kaelbling, M Littman, A Moore, Reinforcement learning: a survey, J. Artif. Intell. Res. 4, 237-285 (1996)

doi:10.1186/1687-1499-2012-337

Cite this article as: Ruiz-Avilés et al.: Traffic steering by self-tuning controllers in enterprise LTE femtocells. EURASIP Journal on Wireless Communications and Networking 2012 2012:337.

\section{Submit your manuscript to a SpringerOpen ${ }^{\mathcal{O}}$ journal and benefit from:}

- Convenient online submission

- Rigorous peer review

- Immediate publication on acceptance

- Open access: articles freely available online

- High visibility within the field

- Retaining the copyright to your article

Submit your next manuscript at $\gg$ springeropen.com 